\title{
Modeling Uncertainty in a Decision Problem by Externalizing Information
}

\author{
I. Parpucea, B. Pârv, T. Socaciu
}

\section{Ilie Parpucea}

Babes-Bolyai University

Faculty of Economic Sciences and Business Administration

Romania, 400591 Cluj-Napoca, 58-60 Teodor Mihali

E-mail: ilie.parpucea@econ.ubbcluj.ro

\section{Bazil Pârv}

Babes-Bolyai University

Faculty of Mathematics and Computer Science

Romania, 400084 Cluj-Napoca, 1 M. Kogălniceanu

E-mail: bparv@cs.ubbcluj.ro

\section{Tiberiu Socaciu}

Stefan cel Mare University

Faculty of Economic Sciences and Public Administration

Romania, 720229 Suceava, 13 Universităţii

E-mail: socaciu@seap.usv.ro

\begin{abstract}
This paper deals with decision problems under uncertainty. The solution of a decision problem involves observation, processing, and modeling of statistical data in order to quantify the uncertainty. Better data measurement and estimation of uncertainty add more consistency to the solution of a decision problem. The paper proposes a new way of predicting the BayesianNash equilibrium which uses information sources to measure new information received by information consumers. Thus, the estimation of uncertainty is based on a more solid mathematical foundation, needed (as in the case of artificial intelligence) to produce logical inferences. From another perspective, the externalization of information helps the software designers to produce better software architectures for decision support systems. An theoretical example illustrates a market situation with a small number of firms, each firm's output being likely to have a large impact on the market price.

Keywords: Bayesian-Nash equilibrium, information source, conditional probability distribution.
\end{abstract}

\section{Introduction}

Game theory, founded by von Neumann and Morgenstern (1947), studies situations in which multiple agents or players interact in order to each maximize an objective (payoff) function. The payoff function of a player is determined not only by its own actions, but also by the actions of other players. In a game with incomplete information, the payoffs also depend on information that is private to the individual agents. This information is known as an agent's type.

Bayesian decision theory is concerned with the question of how a player (decision maker) should choose a particular action from a set of possible choices if the outcome of the choice also depends on some unknown state (from the states of the world). In our approach, the decision maker is modeling the information received by the system (i.e. new information) as an information source ( [2]). A decision problem involves one or several information sources. We 
assume that each person is able to represent his beliefs, as the likelihood of the different $n$ states of the information source, by a subjective discrete probability distribution ( [5]).

The structure of this paper is as follows. After this introductory section, the next one introduces the teoretical background related to games with incomplete information and information sources. The third section presents the Bayes-Nash equilibrium in the presence of information sources, and the fourth discusses a market-related example. The last section compares the proposed approach with the classical one, and outlines future work.

\section{Theoretical background}

This section introduces the main concepts discussed in this paper. It starts with some definitions stating the context, and then defines the concept of information source, both taken from [1] and [3]. The last sub-section introduces the new concept of Bayesian-Nash equilibrium based on information sources.

In what follows, information means a message about an event that has occurred, will occur, or is likely to occur. The received information regarding a possible realization of an event is extremely important. Information is a particular case of reflection, as an interaction between two processes; one's properties (the process that generates or produces information) will be reproduced in another process or several other processes (that consume information). Interaction between two or more processes involves an exchange of information.

\subsection{Games with incomplete information}

Definition 1. A game with incomplete information ( [1]), is denoted by:

$$
\Gamma_{t}=\left(I,\left(F_{i}\right)_{i \in I},\left(p_{t}^{i}(f, \theta)\right)_{i \in I},\left(\Theta_{i}\right)_{i \in I}, \mu_{t}\right)
$$

where:

- $I$ is the set of players, $|I|=m$,

- $F_{i}$ is the strategy set for player $i, i=\overline{1, m}$, and $F=F_{1} \times F_{2} \times \cdots \times F_{m}$ is the the set of all possible strategy profiles;

- $f=\left(f_{1}, f_{2}, \cdots, f_{m}\right) \in F$ is a joint strategy or strategy profile;

- $\Theta_{i}$ is the set of types for the player $i$, and $\Theta=\Theta_{1} \times \Theta_{2} \times \cdots \times \Theta_{m}$ is the joint type space;

- $\theta=\left(\theta_{1}, \theta_{2}, \cdots, \theta_{m}\right) \in \Theta$ is the joint type of all players;

- $p_{t}^{i}(f, \theta)$ is the payoff function for player $i$ at the moment $t$ if the strategy $f$ and the type combination $\theta$ are chosen. Note that the payoff for the player $i$ may depend not only on its type $\theta_{i}$, but also on the other players' type, denoted by $\theta_{-i}$.

- $\mu_{t}$ - the probability distribution on the set $\Theta$ at the moment $t$.

In our exposition, we assume that type sets $\Theta_{i}$ are finite; consequently, $\Theta$ is a finite set also. $\mu_{t}(\theta), \theta \in \Theta$ denotes the probability of chosing type combination $\theta$ at the moment $t$. As in [4], we assume, without loss of generality, that players have incomplete information about their opponents' payoffs but have complete information about the strategies of all other players.

The following definition, taken from [1,3], introduces the classical Bayes-Nash equilibrium of a game with incomplete information. 
Definition 2. A strategy profile $f(\theta)=\left(f_{1}\left(\theta_{1}\right), f_{2}\left(\theta_{2}\right), \cdots, f_{m}\left(\theta_{m}\right)\right)$ constitutes a Bayes-Nash equilibrium of a game $\Gamma_{t}$ with incomplete information if the following inequality:

$$
\sum_{\theta_{-i} \in \Theta_{-i}} p_{t}^{i}\left(f_{i}^{*}\left(\theta_{i}\right), f_{-i}^{*}\left(\theta_{-i}\right), \theta_{i}, \theta_{-i}\right) \mu_{t}\left(\theta_{-i} \mid \theta_{i}\right) \geq \sum_{\theta_{-i} \in \Theta_{-i}} p_{t}^{i}\left(f_{i}\left(\theta_{i}\right), f_{-i}^{*}\left(\theta_{-i}\right), \theta_{i}, \theta_{-i}\right) \cdot \mu_{t}\left(\theta_{-i} \mid \theta_{i}\right)
$$

holds for all possible players $i \in I$ and all types $\theta_{i} \in \Theta_{i}$ and all strategies $f_{i} \in F_{i}$.

\subsection{Information sources}

A process (information producer or consumer) is specified by a set of $n$ variables, denoted by $V=\left\{V_{1}, V_{2}, \cdots, V_{n}\right\}$, where $V_{j}$ is the set of values for the $j^{\text {th }}$ variable: $V_{j}=\left\{v_{j}^{1}, v_{j}^{2}, \cdots, v_{j}^{n_{j}}\right\}$. The state of a process at a certain moment is given by the vector $v=\left\{v_{1}, v_{2}, \cdots, v_{n}\right\}, v_{j} \in$ $V_{j}, j=\overline{1, n}$. Future values of all variables are random, and the realization of the states depends on received information. This information is in message form, decreasing or increasing the uncertainty of the realization of an event.

An information source is a way of specifying the states of a process, regarding one or several variables. The information source assigned to the $j^{\text {th }}$ variable is denoted by $S^{j}$, and the set of distinct values $v_{j}^{k} \in V_{j}, k=\overline{1, n_{j}}$, represents a complete space of events. The simultaneous realization of two events is impossible, and the union of the events represents a certain event. A state $s_{j}^{k}$ of the information source $S^{j}$ is assigned to each event $v_{j}=v_{j}^{k}$. For a bounded interval of time, only information sources with a finite number of states will be taken into account.

Consider the following assumptions:

- each player is able to represent his beliefs, as to the likelihood of the different $n_{j}$ states of the information source $S^{j}$, by a subjective discrete probability distribution.

- the information source $S^{j}$ has discrete states and the individual is supposed to be able to assign to each state $s_{j}^{k}$ a degree of belief, in the form of (normalized) numerical weights $p_{j}^{k}$, between zero and one and whose sum is one:

$\forall j=\overline{1, n}: 0 \leq p_{j}^{k} \leq 1, \forall k=\overline{1, n_{j}} ; \sum_{k=1}^{n_{j}} p_{j}^{k}=1$.

( $p_{j}^{k}$ is the probability that the state $s_{j}^{k}$ occurs).

If the information source $S^{j}$ has $n_{j}$ states, the set of states and probabilities defined at a moment $t$, forms a discrete random variable denoted by:

$$
S_{t}^{j}:\left(\begin{array}{c}
s_{j}^{k} \\
p_{j}^{k}(t)
\end{array}\right)_{k=\overline{1, n_{j}}}, j=\overline{1, n} .
$$

A simple information source is an information source defined with respect to a single variable $V_{j}$. A complex information source is an information source defined with respect to two or more variables, which can be independent or dependent. In the second case (i.e. the variables are related to one another), the mathematical model of the complex information source needs to contain this dependency.

In order to illustrate how a complex information source is constructed, let's consider for the beginning the simplest case of two independent variables, $V_{1} \in V$ and $V_{2} \in V$ and assign to each variable a simple information source, $S_{t}^{1}$ and $S_{t}^{2}$ respectively:

$$
S_{t}^{1}:\left(\begin{array}{c}
s_{1}^{k} \\
p_{1}^{k}(t)
\end{array}\right)_{k=\overline{1, n_{1}}}, S_{t}^{2}:\left(\begin{array}{c}
s_{2}^{l} \\
p_{2}^{l}(t)
\end{array}\right)_{l=\overline{1, n_{2}}},
$$

where $s_{1}^{k}, k=\overline{1, n_{1}}$ are the states of information source $S_{t}^{1}$ and $s_{2}^{l}, l=\overline{1, n_{2}}$ are the states of information source $S_{t}^{2}$. 
The complex information source $S C_{t}^{1,2}$, built with respect to the variables $V_{1}$ and $V_{2}$ at the moment $t$, has the following mathematical model:

$$
S C_{t}^{1,2}:\left(\begin{array}{c}
s_{1}^{k} s_{2}^{j} \\
p_{1}^{k}(t) p_{2}^{j}(t)
\end{array}\right)_{k=\overline{1, n_{1}}, j=\overline{1, n_{2}}} .
$$

Now let us consider the case of two dependent variables, with the simple information source $S_{t}^{1}$ assigned to the independent variable $V_{1}$, and the simple information source $S_{t}^{2}$ assigned to the dependent variable $V_{2}$. The discrete random variable $S_{t}^{1}$ is:

$$
S_{t}^{1}:\left(\begin{array}{c}
s_{1}^{k} \\
p_{1}^{k}(t)
\end{array}\right)_{k=\overline{1, n_{1}}} .
$$

For a state of the source $S_{t}^{1}$, denoted by $s_{1}^{k}$, the information source $S_{t}^{2}$ conditioned by the state $s_{1}^{k}$ is defined as follows:

$$
S_{t}^{2 / 1}\left(s_{1}^{k}\right):\left(\begin{array}{c}
s_{2}^{l} \\
p_{2}^{k, l}(t)
\end{array}\right)_{l=\overline{1, n_{2}}},
$$

where $p_{2}^{k, l}(t)=P\left(S_{t}^{2}=s_{2}^{l} \mid S_{t}^{1}=s_{1}^{l}\right)$ is the probability of occurence of state $s_{2}^{l}$ conditioned by state $s_{1}^{k}$.

The complex information source $S C_{t}^{2 / 1}$, constructed by considering the two variables, has the following form:

$$
S C_{t}^{2 / 1}:\left(\begin{array}{c}
s_{1}^{k} s_{2}^{l} \\
p_{1}^{k}(t) \cdot p_{2}^{k, l}(t)
\end{array}\right)_{k=\overline{1, n_{1}}, l=\overline{1, n_{2}}},
$$

where the probability of occurence of the state $s_{1}^{k} s_{2}^{l}$ is:

$P\left(S_{t}^{1}=s_{1}^{k} ; S_{t}^{2}=s_{2}^{l}\right)=P\left(S_{t}^{1}=s_{1}^{k}\right) \cdot P\left(S_{t}^{2}=s_{2}^{l} \mid S_{t}^{1}=s_{1}^{k}\right)=p_{1}^{k}(t) \cdot p_{2}^{k, l}(t)$.

If $S_{t}^{2}$ is a discrete probability distribution and $S_{t}^{1}$ is an information source, then, according to the above discussion, we can say that $S_{t}^{2}$ is a distribution conditioned by information source $S_{t}^{1}$. Therefore we have a probability distribution updated by an information source.

\section{Bayes-Nash equilibrium in the presence of information sources}

Let us consider now the probability distribution $\mu_{t}$ defined on the discrete set $\Theta$ and a information source $S_{t}$ (simple or complex), common to all players. According to the probability distribution conditioned by an information source discussed above, we have:

$$
P\left(\mu_{t}=\theta ; S_{t}=s^{j}\right)=P\left(S_{t}=s^{j}\right) \cdot P\left(\mu_{t}=\theta \mid S_{t}=s^{j}\right), \text { where } \theta \in \Theta \text { and } s^{j} \in S_{t} .
$$

\subsection{Notations}

Considering the following notations:

- $P\left(\mu_{t}, S_{t}\right)$ - the joint probability of $\mu_{t}$ and $S_{t}$ occurring simultaneously, referred to as the historic probability distribution,

- $P\left(\mu_{t} / S_{t}\right)$ - the probability of $\mu_{t}$ occurrring conditional on $S_{t}$ having occurred (i.e. the conditional probability of $\mu_{t}$ given $S_{t}$ ), also known as the probability distribution of information source, and

- $P\left(S_{t}\right)$ - the marginal probability of $S_{t}$, referred to as the posterior probability distribution,

the following equation holds (as in [7]): $P\left(\mu_{t} / S_{t}\right)=\frac{P\left(\mu_{t}, S_{t}\right)}{P\left(S_{t}\right)}$.

According to the above, posterior means historical updated with information, i.e. the probability distribution $\mu_{t}$ conditioned by the information source $S_{t}$, denoted by $\mu c_{t}$, is the probability distribution $\mu_{t}$ updated by the information source $S_{t}$. 
The information source $S_{t}$ is a possible probability distribution of states at the moment $t+1$. Denoting the game with incomplete information at the moment $t+1$ and based on $S_{t}$ with $\Gamma_{t+1}$, it can be defined recursively as follows:

$\Gamma_{t+1}=\Gamma_{t}\left(S_{t}\right)$, where $\Gamma_{t+1}=\left(I,\left(F_{i}\right)_{i \in I},\left(p_{t}^{i}(f, \theta)\right)_{i \in I},\left(\Theta_{i}\right)_{i \in I}, \mu c_{t}\right)$.

The game $\Gamma_{t+1}$ is the updated game $\Gamma_{t}$ based upon $S_{t}$. This information source updates probability distribution $\mu_{t}$ on $\Theta$ and thus the equilibrium of $\Gamma_{t}$ is modified.

\subsection{Decision functions and strategies}

If a player receives information about his/her own type, then he/she can choose a particular strategy to maximize his/her expected payoff.

Definition 3. A decision function of player $i \in I$, denoted by $f_{i}($.$) , is a function that, for$ each type $\theta_{i} \in \Theta_{i}$, specifies the strategy $f_{i}\left(\theta_{i}\right) \in F_{i}$ this player will choose if his/her type turns out to be $\theta_{i}$.

Let $\mu c_{t}^{j}\left(\theta_{-i} \mid \theta(i)\right)$ be the updated probability obtained by using Bayesian updating rule, of a particular type combination for the opponents $\theta_{-i}$, given that player $i$ has type $\theta_{i}$. For each type profile $\theta \in \Theta$, there are updated beliefs for each player, i.e. a list of conditional probability distributions $\left(\mu c_{t}^{1}\left(\theta_{-1} \mid \theta_{1}\right), \cdots, \mu c_{t}^{m}\left(\theta_{-m} \mid \theta_{m}\right)\right)$. Players' beliefs after they have received information about their types, are no longer identical.

Definition 4. The strategy combination of all players except player $i$, that will be played according to the decision functions $f_{-i}($.$) , if type combination \theta_{-i}$ occurs, is a list of decision functions $f_{-i}()=.\left(f_{1}(),. \cdots, f_{i-1}(),. f_{i+1}(),. \cdots, f_{I}().\right)$ for all players (other than player $i$ ) and $\theta_{-i}()=.\left(\theta_{1}, \cdots, \theta_{i-1}, \theta_{i+1}, \cdots, \theta_{I}\right)$, a type combination for the other players, $f_{-i}\left(\theta_{-i}\right)$, that is: $f_{-i}\left(\theta_{i}\right)=\left(f_{1}\left(\theta_{1}\right), \cdots, f_{i-1}\left(\theta_{i-1}\right), f_{i+1}\left(\theta_{i+1}\right), \cdots, f_{m}\left(\theta_{m}\right)\right)$.

\subsection{Bayes-Nash equilibrium in the presence of information sources}

The above definitions allow us to give the following:

Definition 5. The Bayes - Nash equilibrium of the game $\Gamma_{t+1}$ is a list of decision functions $\left(f_{1}^{*}(),. \cdots, f_{I}^{*}().\right)$, such that for all possible players $i \in I$ and all types $\theta_{i} \in \Theta_{i}$ :

$$
\sum_{\theta_{-i} \in \Theta_{-i}} p_{t}^{i}\left(f_{i}^{*}\left(\theta_{i}\right), f_{-i}^{*}\left(\theta_{-i}\right), \theta_{i}, \theta_{-i}\right) \cdot \mu c_{t}\left(\theta_{-i} \mid \theta_{i}\right) \geq \sum_{\theta_{-i} \in \Theta_{-i}} p_{t}^{i}\left(f_{i}, f_{-i}^{*}\left(\theta_{-i}\right), \theta_{i}, \theta_{-i}\right) \cdot \mu c_{t}^{i}\left(\theta_{-i} \mid \theta_{i}\right)
$$

holds for all strategies $f_{i} \in F_{t}$.

The equilibrium of $\Gamma_{t+1}$ can differ from the equilibrium of $\Gamma_{t}$ due to the information in $S_{t}$. For a given player $i \in I$ the updated equilibrium of $\Gamma_{t+1}$ is:

$$
f_{i}^{*}(.)=\sum_{j} f_{i j}^{*}(.) \cdot p^{j}(t)
$$

where $f_{i j}^{*}($.$) is the equilibrium of player i$ for the state $s^{j}$ of the information source $S_{t}$ and $p^{j}(t)$ is the probability that $S_{t}=s^{j}$. The above equation represents the updated equilibrium as a weighted average of all equilibria for the states of information source.

\section{A market-related example}

Consider a game with incomplete information, where the players are two firms supplying slightly different products (produced with zero production costs, as in [1]), with prices denoted 
by $p_{1}$ and $p_{2}$. As a result of new received information, the variation of each price around the average price can be modeled using two simple information sources, $S_{t}^{1}$ and $S_{t}^{2}$, as it follows:

$$
S_{t}^{1}:\left(\begin{array}{cc}
s_{1}^{1}: p_{1} \leq \overline{p_{1}} & s_{1}^{2}: p_{1}>\overline{p_{1}} \\
\pi_{1} & 1-\pi_{1}
\end{array}\right) ; S_{t}^{2}:\left(\begin{array}{cc}
s_{2}^{1}: p_{2} \leq \overline{p_{2}} & s_{2}^{2}: p_{2}>\overline{p_{2}} \\
\pi_{2} & 1-\pi_{2}
\end{array}\right)
$$

where $\overline{p_{1}}$ represents the average price of the first firm, and $\overline{p_{2}}$ the average price of the second firm. Both information sources have two states, $\left(s_{1}^{1}, s_{1}^{2}\right)$ and $\left(s_{2}^{1}, s_{2}^{2}\right)$, with the probabilities $\left(\pi_{1}, 1-\pi_{1}\right)$ and $\left(\pi_{2}, 1-\pi_{2}\right)$, respectively.

\subsection{Notations}

For our example, consider the following problem-specific notations:

- The demand functions for the goods of the two firms:

$d_{1}\left(p_{1}, p_{2}\right)=a \cdot \Delta p_{1}+b \cdot \Delta p_{2}, d_{2}\left(p_{1}, p_{2}\right)=c \cdot \Delta p_{1}+d \cdot \Delta p_{2}$,

where $\Delta p_{i}=p_{i}-\overline{p_{i}}, i=\overline{1,2}$, are the deviation of price $p_{i}$ from the average price $\overline{p_{i}}$. Firm one does not know parameters $c$ and $d$; firm two does not know parameters $a$ and $b$.

- The sets of possible types of the two players:

$$
\Theta_{1}=\left\{\left(a_{i}, b_{j}\right), i=1,2 ; j=1,2\right\}, \Theta_{2}=\left\{\left(c_{i}, d_{j}\right), i=1,2 ; j=1,2\right\} \text {; }
$$

- The payoff functions of the two players:

$$
\begin{aligned}
\Pi_{1}\left(p_{1}, p_{2},\left(a_{i}, b_{j}\right)\right) & =\left(a_{i} \cdot \Delta p_{1}+b_{j} \cdot \Delta p_{2}\right) \cdot p_{1}, \\
\Pi_{2}\left(p_{1}, p_{2},\left(c_{i}, d_{j}\right)\right) & =\left(c_{i} \cdot \Delta p_{1}+d_{j} \cdot \Delta p_{2}\right) \cdot p_{2} .
\end{aligned}
$$

\subsection{Building the complex information source}

With the above notations, the complex information source is rewritten as:

$$
S C_{t+1}:\left(\begin{array}{cccc}
p_{1} \leq \overline{p_{1}} \wedge p_{2} \leq \overline{p_{2}} & p_{1} \leq \overline{p_{1}} \wedge p_{2}>\overline{p_{2}} & p_{1}>\overline{p_{1}} \wedge p_{2} \leq \overline{p_{2}} & p_{1}>\overline{p_{1}} \wedge p_{2}>\overline{p_{2}} \\
\pi_{1} \cdot \pi_{2} & \pi_{1} \cdot\left(1-\pi_{2}\right) & \left(1-\pi_{1}\right) \cdot \pi_{2} & \left(1-\pi_{1}\right) \cdot\left(1-\pi_{2}\right)
\end{array}\right)
$$

no matter what dependency relation is between the two prices considered. The information source $S C_{t+1}$ describes the behavior of the market of the both products considering the variation of their prices in the next period, as a result of the received information.

In a Bayesian-Nash equilibrium, each firm is supposed to choose a type contingent strategy, that is decision functions $p_{1}($.$) and p_{2}($.$) respectively, which is the best response to the oppo-$ nent's decision function. In this example, $\mu c_{t}$ is a probability distribution defined on $\Theta_{1} \times \Theta_{2}$ and conditioned by the information source $S C_{t}$. For a state $s_{1}^{k} s_{2}^{l}$ of source $S C_{t}$ we build two conditional distributions, $\mu c_{t}^{1}$ and $\mu c_{t}^{2}$ as can be seen next.

\subsection{Computing the best response for the firm one}

Consider $p_{2}()=.\left(p_{2}\left(c_{1}, d_{1}\right), p_{2}\left(c_{1}, d_{2}\right), p_{2}\left(c_{2}, d_{1}\right), p_{2}\left(c_{2}, d_{2}\right)\right)$ as given (fixed) and suppose that firm one has just learned that it has the demand parameters $\left(a_{1}, b_{1}\right)$. Firm one's expected payoff can be rewritten as:

$$
\begin{array}{r}
\Pi_{1}\left(p_{1}\left(a_{1}, b_{1}\right), p_{2}\left(c_{1}, d_{1}\right)\right) \cdot \mu c_{t}^{1}\left(\left(c_{1}, d_{1}\right) \mid\left(a_{1}, b_{1}\right)\right)+ \\
+\Pi_{1}\left(p_{1}\left(a_{1}, b_{2}\right), p_{2}\left(c_{1}, d_{2}\right)\right) \cdot \mu c_{t}^{1}\left(\left(c_{1}, d_{2}\right) \mid\left(a_{1}, b_{1}\right)\right)+ \\
+\Pi_{1}\left(p_{1}\left(a_{2}, b_{1}\right), p_{2}\left(c_{2}, d_{1}\right)\right) \cdot \mu c_{t}^{1}\left(\left(c_{2}, d_{1}\right) \mid\left(a_{1}, b_{1}\right)\right)+ \\
+\Pi_{1}\left(p_{1}\left(a_{2}, b_{2}\right), p_{2}\left(c_{2}, d_{2}\right)\right) \cdot \mu c_{t}^{1}\left(\left(c_{2}, d_{2}\right) \mid\left(a_{1}, b_{1}\right)\right)= \\
=a_{1} \cdot p_{1}^{2}\left(a_{1}, b_{1}\right)+p_{1}\left(a_{1}, b_{1}\right)\left(b_{1} \cdot \overline{p_{2}}\left(c_{i} d_{j} \mid a_{1} b_{1}\right)-a_{1} \overline{p_{1}}-b_{1} \overline{p_{2}}\right) .
\end{array}
$$


In the above equation we used the following notation for the average price $\overline{p_{2}}$ conditioned by state $\left(a_{1}, b_{1}\right)$ :

$$
\begin{aligned}
\left.\overline{p_{2}}\left(c_{i}, d_{j}\right) \mid a_{1} b_{1}\right)= & p_{2}\left(c_{1}, d_{1}\right) \cdot \mu c_{t}^{1}\left(\left(c_{1}, d_{1}\right) \mid\left(a_{1}, b_{1}\right)\right)+p_{2}\left(c_{1}, d_{2}\right) \cdot \mu c_{t}^{1}\left(\left(c_{1}, d_{2}\right) \mid\left(a_{1}, b_{1}\right)\right)+ \\
& +p_{2}\left(c_{2}, d_{1}\right) \cdot \mu c_{t}^{1}\left(\left(c_{2}, d_{1}\right) \mid\left(a_{1}, b_{1}\right)\right)+p_{2}\left(c_{2}, d_{2}\right) \cdot \mu c_{t}^{1}\left(\left(c_{2}, d_{2}\right) \mid\left(a_{1}, b_{1}\right)\right) .
\end{aligned}
$$

The payoff function (4) is continuously differentiable in firm one's strategy $p_{1}($.$) . Therefore,$ any $p_{1}$ satisfying the first - order condition for a maximum will be the best response to the type-contingent strategy $p_{2}($.$) , previously considered. Solving the first - order condition for the$ maximum of the expected payoff function (4), one obtains the following best response $p_{1}^{*}\left(a_{1}, b_{1}\right)$ for firm one of type $\left(a_{1}, b_{1}\right)$ to the decision functions $\left(p_{2}\left(c_{1}, d_{1}\right), p_{2}\left(c_{2}, d_{2}\right), p_{2}\left(c_{2}, d_{1}\right), p_{2}\left(c_{2}, d_{2}\right)\right)$ of firm two:

$$
p_{1}^{*}\left(a_{1}, b_{1}\right)=\frac{1}{2}\left(\overline{p_{1}}+\frac{b_{1}}{a_{1}}\left(\overline{p_{2}}-\overline{p_{2}}\left(c_{i} d_{j} \mid a_{1} b_{1}\right)\right)\right) .
$$

In a similar way, one obtains for all other types of firm one:

$$
\begin{aligned}
p_{1}^{*}\left(a_{1}, b_{2}\right) & =\frac{1}{2}\left(\overline{p_{1}}+\frac{b_{2}}{a_{1}}\left(\overline{p_{2}}-\overline{p_{2}}\left(c_{i} d_{j} \mid a_{1} b_{2}\right)\right)\right), \\
p_{1}^{*}\left(a_{2}, b_{1}\right) & =\frac{1}{2}\left(\overline{p_{1}}+\frac{b_{1}}{a_{2}}\left(\overline{p_{2}}-\overline{p_{2}}\left(c_{i} d_{j} \mid a_{2} b_{1}\right)\right)\right), \\
p_{1}^{*}\left(a_{2}, b_{2}\right) & =\frac{1}{2}\left(\overline{p_{1}}+\frac{b_{2}}{a_{2}}\left(\overline{p_{2}}-\overline{p_{2}}\left(c_{i} d_{j} \mid a_{2} b_{2}\right)\right) .\right.
\end{aligned}
$$

\subsection{Computing the best response for the firm two}

Now consider that firm two learns that its type is $\left(c_{1}, d_{1}\right)$. For a fixed type-contingent strategy of firm one $p_{1}()=.\left(p_{1}\left(a_{1}, b_{1}\right), p_{1}\left(a_{1}, b_{2}\right), p_{1}\left(a_{2}, b_{1}\right), p_{1}\left(a_{2}, b_{2}\right)\right)$, the expected payoff of firm two will be as follows:

$$
\begin{aligned}
& \Pi_{2}\left(p_{1}\left(a_{1}, b_{1}\right), p_{2}\left(c_{1}, d_{1}\right)\right) \cdot \mu c_{t}^{2}\left(\left(a_{1}, b_{1}\right) \mid\left(c_{1}, d_{1}\right)\right)+ \\
&+ \Pi_{2}\left(p_{1}\left(a_{1}, b_{2}\right), p_{2}\left(c_{1}, d_{2}\right)\right) \cdot \mu c_{t}^{2}\left(\left(a_{1}, b_{2}\right) \mid\left(c_{1}, d_{1}\right)\right)+ \\
&+ \Pi_{2}\left(p_{1}\left(a_{2}, b_{1}\right), p_{2}\left(c_{2}, d_{1}\right)\right) \cdot \mu c_{t}^{2}\left(\left(a_{2}, b_{1}\right) \mid\left(c_{1}, d_{1}\right)\right)+ \\
&+ \Pi_{2}\left(p_{1}\left(a_{2}, b_{2}\right), p_{2}\left(c_{2}, d_{2}\right)\right) \cdot \mu c_{t}^{2}\left(\left(a_{2}, b_{2}\right) \mid\left(c_{1}, d_{1}\right)\right)= \\
&=d_{1} \cdot p_{2}^{2}\left(c_{1}, d_{1}\right)+p_{2}\left(c_{1}, d_{1}\right)\left(c_{1} \cdot \overline{p_{1}}\left(a_{i} b_{j} \mid c_{1} d_{1}\right)-c_{1} \overline{p_{1}}-d_{1} \overline{p_{2}}\right) .
\end{aligned}
$$

In the equation (9) of the payoff function for the second firm, the average price $\overline{p_{1}}$ conditioned by the state $\left(c_{1}, d_{1}\right)$, is given by:

$$
\begin{array}{r}
\left.\overline{p_{1}}\left(a_{i}, b_{j}\right) \mid c_{1} d_{1}\right)=p_{1}\left(a_{1}, b_{1}\right) \cdot \mu c_{t}^{2}\left(\left(a_{1}, b_{1}\right) \mid\left(c_{1}, d_{1}\right)\right)+p_{1}\left(a_{1}, b_{2}\right) \cdot \mu c_{t}^{2}\left(\left(a_{1}, b_{2}\right) \mid\left(c_{1}, d_{1}\right)\right)+ \\
p_{1}\left(a_{2}, b_{1}\right) \cdot \mu c_{t}^{2}\left(\left(a_{2}, b_{1}\right) \mid\left(c_{1}, d_{1}\right)\right)+p_{1}\left(a_{2}, b_{2}\right) \cdot \mu c_{t}^{2}\left(\left(a_{2}, b_{2}\right) \mid\left(c_{1}, d_{1}\right)\right) .
\end{array}
$$

First-order condition gives the best response function for a firm of type $\left(c_{1}, d_{1}\right)$ :

$$
p_{2}^{*}\left(c_{1}, d_{1}\right)=\frac{1}{2}\left(\overline{p_{2}}+\frac{c_{1}}{d_{1}}\left(\overline{p_{1}}-\overline{p_{1}}\left(a_{i} b_{j} \mid c_{1} d_{1}\right)\right)\right) .
$$

A similar calculation yields firm two's best response for all other types of the following typecontingent strategies:

$$
\begin{aligned}
& p_{2}^{*}\left(c_{1}, d_{2}\right)=\frac{1}{2}\left(\overline{p_{2}}+\frac{c_{2}}{d_{1}}\left(\overline{p_{1}}-\overline{p_{1}}\left(a_{i} b_{j} \mid c_{1} d_{2}\right)\right)\right), \\
& p_{2}^{*}\left(c_{2}, d_{1}\right)=\frac{1}{2}\left(\overline{p_{2}}+\frac{c_{1}}{d_{2}}\left(\overline{p_{1}}-\overline{p_{1}}\left(a_{i} b_{j} \mid c_{2} d_{1}\right)\right)\right), \\
& p_{2}^{*}\left(c_{2}, d_{2}\right)=\frac{1}{2}\left(\overline{p_{2}}+\frac{c_{2}}{d_{2}}\left(\overline{p_{1}}-\overline{p_{1}}\left(a_{i} b_{j} \mid c_{2} d_{2}\right)\right)\right) .
\end{aligned}
$$




\subsection{Conclusion}

In order to find a Bayesian-Nash equilibrium, one has to solve the system of equations given by the best response functions. With two players and four types for each player, this leads to a system of eight equations. The solution, $\left(p_{1}^{*}(),. p_{2}^{*}().\right)$, is the Bayesian -Nash equilibrium. The probability of realization of equilibrium prices $\left(p_{1}^{*}(),. p_{2}^{*}().\right)$, as a result of information received, is equal to the probability of realization of state $s_{1}^{k} s_{2}^{l}$ of complex source $S C_{t+1}$.

\section{Conclusions and Future Works}

\subsection{Our approach vs other approaches}

The main points of our approach are as follows:

- The complexity of uncertainty is given by the great (huge) number of variables; when the complexity of a decision problem (and the number of components dominated by uncertainty) grows, it is recommended to use a Bayesian network ( [6]); in our case, we use a simple Bayesian network, subject to a learning algorithm;

- The original idea is to separate the set of problem components into two disjoint subsets: (a) deterministic components, and (b) components dominated by uncertainty; the separation of game information into external and internal can be done for each decision problem dominated by uncertainty;

- This separation allows you to study the influence of each individual factor to the solution of the game in a more efficient way; also, it suggests some architectural patterns (styles) to be used when designing a decision support system. The paper [8] discusses this issue in more detail.

The essential difference between the classic approach and those proposed in this paper is given by the separation of the information external to the game from the game-specific information. This separation follows the separation of responsibilities principle. This way, both external and internal elements of the game are easier to model and understand.

The classical approach does not make any difference between these two categories of information; more precisely, the influence of external information on the uncertainty that dominates the game is not taken into account/quantified. By splitting the game information into external and internal, the former being modeled by information sources, the influence of external environment on the variation of the solution is better captured and quantified. This provides a better evaluation of the contribution of individual factors to the predicted equilibrium.

Another advantage of this separation is that it allows a better, easier calibration of the model, by comparing the computed equilibrium with real solution, taken from historical data.

\subsection{Future work}

Our future efforts are directed to apply this general algorithm to various games with incomplete information and to build decision support systems based on it.

\section{Acknowledgements}

This work was supported by the grant ID_2586, sponsored by NURC - Romanian National University Research Council (CNCSIS). 


\section{Bibliography}

[1] Eichberger, I., Game Theory for Economists, Academic Press, 1993.

[2] Florea, I., Parpucea, I., Economic Cybernetics (Romanian), Babeş-Bolyai University, 1993.

[3] Fudenberg, D., Tirole, J., Game Theory, MIT Press, 1991.

[4] Harsanyi, J.C., Games with Incomplete Information Played by Bayesian Players, Parts I, II, III, Management Science, 14, 1967, pp. 159-182, 320-334, 486-502.

[5] Hirshleifer ,J., Riley J. G., The Analytics of Uncertainty and Information, Cambridge University Press, 1995.

[6] Jensen, F.V., Nielsen, T.D., Bayesian Networks and Decision Graphs, Springer, 2nd ed., 2007

[7] Koop, G., Bayesian Econometrics, Wiley, 2003.

[8] Pârv, B., Parpucea, I., Computing Bayes-Nash Equilibrium for Games with Incomplete Information by Using Information Sources, submitted to IJCCC.

[9] Reiz, B., Csato, L. Tree-Like Bayesian Network Classifiers for Surgery Survival Chance Prediction, Int. J. of Computers, Communications 83 Control, III, 2008, pp. 470-474. 